\title{
On two-valued measure and double statistical convergence in 2-normed spaces
}

Ekrem Savaş*

Dedicated to Professor Hari M Srivastava

"Correspondence:

ekremsavas@yahoo.com; esavas@iticu.edu.tr

Department of Mathematics, Istanbul Ticaret University, Üsküdar, Istanbul, Turkey

\begin{abstract}
In this paper we introduce some new double difference lacunary sequence spaces using Orlicz functions, generalized double difference sequences and a two-valued measure $\mu$ in 2-normed spaces, and we also examine some of their properties.

MSC: Primary 40H05; secondary 40C05
\end{abstract}

Keywords: statistical convergence; $\mu$-statistical convergence; 2-normed space; paranormed space; sequence spaces; double lacunary sequence

\section{Introduction}

The notion of summability of single sequences with respect to a two-valued measure was introduced by Connor $[1,2]$ as a very interesting generalization of statistical convergence which was defined by Fast [3]. Over the years, and under different names, statistical convergence was discussed in the theory of Fourier analysis, ergodic theory and number theory. Later on, it was further investigated from the sequence spaces point of view and linked with summability theory by Fridy [4], Salat [5]. The notion of statistical convergence was further extended to double sequences independently by Móricz [6] and Mursaleen et al. [7]. Savaş [8] studied statistical convergence in random 2-normed space. For more recent developments on double sequences one can consult the papers (see [9-16]) where more references can be found. In particular, very recently Das and Bhunia investigated the summability of double sequences of real numbers with respect to a twovalued measure and made many interesting observations [17]. In [18], Das and Savaş et al. introduced some generalized double difference sequence spaces using summability with respect to a two-valued measure and an Orlicz function in 2-normed spaces which have a unique non-linear structure. The study of Orlicz sequence spaces was initiated with a certain specific purpose in Banach space theory. Lindenstrauss and Tzafriri [19] investigated Orlicz sequence spaces in more detail and they proved that every Orlicz sequence space $l_{M}$ contains a subspace isomorphic to $l_{p}(1 \leq p<\infty)$. The Orlicz sequence spaces are the special cases of Orlicz spaces studied in [20]. Orlicz spaces find a number of useful applications in the theory of nonlinear integral equations. Whereas the Orlicz sequence spaces are the generalization of $l_{p}$-spaces, the $l_{p}$-spaces find themselves enveloped in Orlicz spaces [21].

2013 Savas: licensee Springer. This is an Open Access article distributed under the terms of the Creative Commons Attribution License (http://creativecommons.org/licenses/by/2.0), which permits unrestricted use, distribution, and reproduction in any medium, provided the original work is properly cited. 
The concept of 2-normed spaces was initially introduced by Gahler [22, 23] as a very interesting non-linear extension of the idea of usual normed linear spaces. Some initial studies on this structure can be seen in [22-24]. Recently a lot of interesting developments have occurred in 2-normed spaces in summability theory and related topics (see $[18,25-30])$.

In this paper, in a natural way, we first define statistical convergence for double sequences in 2-normed spaces using a two-valued measure and also prove some interesting theorems. Furthermore, we introduce some new sequence spaces in 2-normed spaces using Orlicz functions, generalized double difference sequences and a two-valued measure $\mu$.

\section{Preliminaries}

Throughout the paper $\mathbb{N}$ denotes the set of all natural numbers, $\chi_{A}$ represents the characteristic function of $A \subseteq \mathbb{N}$ and $\mathbb{R}$ represents the set of all real numbers.

Recall that a set $A \subseteq \mathbb{N}$ is said to have the asymptotic density $d(A)$ if

$$
d(A)=\lim _{n \rightarrow \infty}\left(\frac{1}{n}\right) \sum_{j=1}^{n} \chi_{A}(j)
$$

exists.

Definition 2.1 [3] A sequence $\left\{x_{n}\right\}_{n \in \mathbb{N}}$ of real numbers is said to be statistically convergent to $\xi \in \mathbb{R}$ if for any $\epsilon>0$ we have $d(A(\epsilon))=0$, where $A(\epsilon)=\left\{n \in \mathbb{N}:\left|x_{n}-\xi\right| \geq \epsilon\right\}$.

In [31] the notion of convergence for double sequences was presented by Pringsheim.

A double sequence $x=\left\{x_{k l}\right\}$ of real numbers is said to be convergent to $L \in \mathbb{R}$ if, for any $\epsilon>0$, there exists $N_{\epsilon} \in \mathbb{N}$ such that $\left|x_{k l}-L\right|<\epsilon$ whenever $k, l \geq N_{\epsilon}$. In this case, we write $\lim _{k, l \rightarrow \infty} x_{k l}=L$.

A double sequence $x=\left\{x_{k l}\right\}$ of real numbers is said to be bounded if there exists a positive real number $M$ such that $\left|x_{k l}\right|<M$ for all $k, l \in \mathbb{N}$. That is, $\|x\|_{(\infty, 2)}=\sup _{k, l \in \mathbb{N}}\left|x_{k l}\right|<\infty$.

Let $K \subseteq \mathbb{N} \times \mathbb{N}$ and let $K(k, l)$ be the cardinality of the set $\{(m, n) \in K: m \leq k, n \leq l\}$. If the sequence $\left\{\frac{K(k, l)}{k . l}\right\}_{k, l \in \mathbb{N}}$ has a limit in the Pringsheim's sense, then we say that $K$ has double natural density and is denoted by $d_{2}(K)=\lim _{k, l \rightarrow \infty} \frac{K(k, l)}{k . l}$.

A statistically convergent double sequence of elements of a metric space $(X, \rho)$ is defined essentially in the same way $\left(\rho\left(x_{k l}, \xi\right) \geq \epsilon\right.$ instead of $\left.\left|x_{k l}-\xi\right| \geq \epsilon\right)$.

Throughout the paper $\mu$ will denote a complete $\{0,1\}$-valued finite additive measure defined on algebra $\Gamma$ of subsets of $\mathbb{N} \times \mathbb{N}$ that contains all subsets of $\mathbb{N} \times \mathbb{N}$ that are contained in the union of a finite number of rows and columns of $\mathbb{N} \times \mathbb{N}$ and $\mu(A)=0$ if $A$ is contained in the union of a finite number of rows and columns of $\mathbb{N} \times \mathbb{N}$ (see [18]).

Definition 2.2 [18] A double sequence $x=\left\{x_{k l}\right\}$ of real numbers is said to be $\mu$-statistically convergent to $L \in \mathbb{R}$ if and only if for any $\epsilon>0, \mu\left(\left\{(k, l) \in \mathbb{N} \times \mathbb{N}:\left|x_{k l}-L\right| \geq \epsilon\right\}\right)=0$.

Definition 2.3 [18] A double sequence $x=\left\{x_{k l}\right\}$ of real numbers is said to be convergent to $L \in \mathbb{R}$ in $\mu$-density if there exists an $A \in \Gamma$ with $\mu(A)=1$ such that $\left\{x_{k l}\right\}_{(k, l) \in A}$ is convergent to $L$. 
Definition 2.4 [23] Let $X$ be a real vector space of dimension $d$, where $2 \leq d<\infty$. A 2-norm on $X$ is a function $\|\cdot, \cdot\|: X \times X \rightarrow \mathbb{R}$ which satisfies (i) $\|x, y\|=0$ if and only if $x$ and $y$ are linearly independent; (ii) $\|x, y\|=\|y, x\|$; (iii) $\|\alpha x, y\|=|\alpha|\|x, y\|, \alpha \in \mathbb{R}$; (iv) $\|x, y+z\| \leq\|x, y\|+\|x, z\|$. The ordered pair $(X,\|\cdot, \cdot\|)$ is then called a 2-normed space.

Let $(X,\|\cdot, \cdot\|)$ be a finite dimensional 2-normed space and let $u=\left\{u_{1}, u_{2}, \ldots, u_{d}\right\}$ be the basis of $X$. We can define the norm $\|\cdot, \cdot\|_{\infty}$ on $X$ by $\|\cdot, \cdot\|_{\infty}=\max \left\{\left\|x, u_{i}\right\|: i=1, \ldots, d\right\}$. Also, $\|x-y\|_{\infty}=\max \left\{\left\|x-y, u_{j}\right\|: j=1, \ldots, d\right\}$.

Let $(X,\|\cdot, \cdot\|)$ be any 2 -normed space and let $S^{\prime \prime}(2-X)$ be the set of all double sequences defined over the 2 -normed space $(X,\|\cdot, \cdot\|)$. Clearly $S^{\prime \prime}(2-X)$ is a linear space under addition and scalar multiplication.

Recall in [20] that an Orlicz function $M:[0, \infty) \rightarrow[0, \infty)$ is a continuous, convex and non-decreasing function such that $M(0)=0$ and $M(x)>0$ for $x>0$, and $M(x) \rightarrow \infty$ as $x \rightarrow \infty$.

Note that if $M$ is an Orlicz function, then $M(\lambda x) \leq \lambda$ for all $\lambda$ with $0<\lambda<1$.

In the later stage, different classes of Orlicz sequence spaces were introduced and studied by Parashar and Choudhary [32], Savaş [28-30, 33-38] and many others.

Definition $2.5[18]$ A double sequence $x=\left\{x_{k l}\right\}$ in a 2-normed space $(X,\|\cdot, \cdot\|)$ is said to be convergent to $L$ in $(X,\|\cdot, \cdot\|)$ if for each $\epsilon>0$ and each $z \in X$, there exists $n_{\epsilon} \in \mathbb{N}$ such that $\left\|x_{k l}-L, z\right\|<\epsilon$ for all $i, j \geq n_{\epsilon}$.

Definition 2.6 [18] Let $\mu$ be a two-valued measure on $\mathbb{N} \times \mathbb{N}$. A double sequence $\left\{x_{k l}\right\}$ in a 2-normed space $(X,\|\cdot, \cdot\|)$ is said to be $\mu$-statistically convergent to some point $x$ in $\mathrm{X}$ if for each pre-assigned $\epsilon>0$ and for each $z \in X, \mu(A(z, \epsilon))=0$, where $A(z, \epsilon)=\{(k, l) \in$ $\left.\mathbb{N} \times \mathbb{N}:\left\|x_{k l}-x, z\right\| \geq \epsilon\right\}$.

Definition 2.7 Let $\mu$ be a two-valued measure on $\mathbb{N} \times \mathbb{N}$. A double sequence $\left\{x_{k l}\right\}$ in a 2-normed space $(X,\|\cdot, \cdot\|)$ is said to be $\mu$-statistically Cauchy if for each pre-assigned $\epsilon>0$ and for each $z \in X$, there exist integers $N=N(\varepsilon, z)$ and $M=M(\varepsilon, z)$ such that $\mu(A(z, \epsilon))=0$, where $A(z, \epsilon)=\left\{(k, l) \in \mathbb{N} \times \mathbb{N}:\left\|x_{k l}-x_{N(\varepsilon, z) M(\varepsilon, z)}, z\right\| \geq \epsilon\right\}$.

We first give the following theorem.

Theorem 2.1 Let $\mu$ be a two-valued measure on $\mathbb{N} \times \mathbb{N}$ and two sequences $\left(x_{k l}\right)$ and $\left(y_{k l}\right)$ in 2-normed space $(X,\|\cdot, \cdot\|)$. If $\left(y_{k l}\right)$ is a $\mu$-convergent sequence such that $x_{k l}=y_{k l} a . a .(k, l)$, then $\left(x_{k l}\right)$ is $\mu$-statistically convergent.

Proof Suppose $\mu\left(\left\{(k, l) \in \mathbb{N} \times \mathbb{N}: x_{k l} \neq y_{k l}\right\}\right)=0$ and $\lim _{m, n \rightarrow \infty}\left\|y_{k l}, z\right\|=\|L, z\|$. Then, for every $\epsilon>0$ and $z \in X$,

$$
\begin{aligned}
\left\{(k, l) \in \mathbb{N} \times \mathbb{N}:\left\|x_{k l}-L, z\right\| \geq \varepsilon\right\} \subseteq & \left\{(k, l) \in \mathbb{N} \times \mathbb{N}:\left\|y_{k l}-L, z\right\| \geq \varepsilon\right\} \\
& \cup\left\{(k, l) \in \mathbb{N} \times \mathbb{N}: x_{k l} \neq y_{k l}\right\} .
\end{aligned}
$$

Therefore

$$
\begin{aligned}
& \mu\left\{(k, l) \in \mathbb{N} \times \mathbb{N}:\left\|x_{k l}-L, z\right\| \geq \varepsilon\right\} \leq \mu\left\{(k, l) \in \mathbb{N} \times \mathbb{N}:\left\|y_{k l}-L, z\right\| \geq \varepsilon\right\} \\
& +\mu\left\{(k, l) \in \mathbb{N} \times \mathbb{N}: x_{k l} \neq y_{k l}\right\} .
\end{aligned}
$$


Since

$$
\lim _{m n \rightarrow \infty}\left\|y_{k l}, z\right\|=\|L, z\|
$$

for every $z \in X$, the set

$$
\left\{(k, l) \in \mathbb{N} \times \mathbb{N}:\left\|y_{k l}-L, z\right\| \geq \varepsilon\right\}
$$

contains a finite number of integers. Hence,

$$
\mu\left\{(k, l) \in \mathbb{N} \times \mathbb{N}:\left\|y_{k l}-L, z\right\| \geq \varepsilon\right\}=0 .
$$

We get

$$
\mu\left\{(k, l) \in \mathbb{N} \times \mathbb{N}:\left\|x_{k l}-L, z\right\| \geq \varepsilon\right\}=0
$$

for every $\epsilon>0$ and $z \in X$. Consequently, $\mu$-st- $\lim _{k, l \rightarrow \infty}\left\|x_{k l}, z\right\|=\|L, z\|$. This completes the proof.

Theorem 2.2 Let $\mu$ be a two-valued measure on $\mathbb{N} \times \mathbb{N}$ and let $\left\{x_{k l}\right\}_{k, l \geq 1,1}$ be a $\mu$ statistically Cauchy sequence in a finite dimensional 2 -normed space $(X,\|\cdot, \cdot\|)$. Then there exits a $\mu$-convergent sequence $\left\{y_{k l}\right\}_{k, l \geq 1}$ in $(X,\|\cdot, \cdot\|)$ such that $x_{k l}=y_{k l}$ for a.a. $(k, l)$.

Proof First suppose that $\left\{x_{k l}\right\}_{k, l \geq 1}$ is a statistically Cauchy sequence in $\left(X,\|\cdot, \cdot\|_{\infty}\right)$. Choose natural numbers $M_{1}$ and $N_{1}$ such that the closed ball $B_{u}^{1,1}=B_{u}\left(x_{M_{1} N_{1}}, 1\right)$ contains $x_{k l}$ for a.a. $(k, l)$. Then choose natural numbers $M_{2}$ and $N_{2}$ such that the closed ball $B_{2,2}=$ $B_{u}\left(x_{M_{2} N_{2}}, \frac{1}{(2.2)}\right)$ contains $x_{k l}$ for a.a. $(k, l)$. Note that $B_{u}^{2,2}=B_{u}^{1,1} \cup B_{2,2}$ also contains $x_{k l}$ for a.a. $(k, l)$. Thus, by continuing this process, we can obtain a sequence $\left\{B_{u}^{p, q}\right\}_{p, q \geq 1,1}$ of nested closed balls such that $\operatorname{diam}\left\{B_{u}^{p, q}\right\} \leq \frac{1}{2^{p} 2^{q}}$. Therefore $\bigcap_{p, q=1,1}^{\infty, \infty} B_{u}^{p, q}=\{A\}$. Since each $B_{u}^{p, q}$ contains $x_{k l}$ for a.a. $(k, l)$, we can choose a sequence of strictly increasing natural numbers $\left\{T_{p, q}\right\}_{p, q \geq 1}$ such that

$$
\frac{1}{k l}\left|\left\{(k, l) \in \mathbb{N} \times \mathbb{N}: x_{k l} \notin B_{u}^{p, q}\right\}\right|<\frac{1}{p, q} \quad \text { if } k, l>T_{p, q} .
$$

Hence we have

$$
\mu\left\{(k, l) \in \mathbb{N} \times \mathbb{N}: x_{k l} \notin B_{u}^{p, q}\right\}=0 \quad \text { if } k, l>T_{p, q}
$$

Put $W_{p ; q}=\left\{(k, l) \in \mathbb{N} \times \mathbb{N}:(k, l)>T_{p, q}, x_{k l} \notin B_{u}^{p, q}\right\}$ for all $p, q \geq 1$, and $W=\bigcup_{p, q=1,1}^{\infty, \infty} W_{p, q}$. Now define the sequence $\left\{y_{k l}\right\}_{k l \geq 1}$ as follows:

$$
y_{k l}= \begin{cases}A & \text { if }(k, l) \in W \\ x_{k l} & \text { otherwise }\end{cases}
$$

Note that $\lim _{k, l \rightarrow \infty, \infty} y_{k l}=A$. In fact, for each $\epsilon>0$, choose a natural number $p, q$ such that $\epsilon>\frac{1}{p, q}>0$. Then, for each $k l>T_{p q}$, or $y_{k l}=A$ or $y_{k l}=x_{k l} \in B_{u}^{p, q}$ and so in each case 
$\left\|y_{k l}-A\right\|_{\infty} \leq \operatorname{diam}\left\{B_{u}^{p, q}\right\} \leq \frac{1}{2^{p-1} 2^{q-1}}$. Since $\left\{(k, l) \in \mathbb{N} \times \mathbb{N}: y_{k l} \neq x_{k l}\right\} \subseteq\left\{(k, l) \in \mathbb{N} \times \mathbb{N}: x_{k l} \notin\right.$ $\left.B_{u}^{p, q}\right\}$, we have

$$
\mu\left\{(k, l) \in \mathbb{N} \times \mathbb{N}: y_{k l} \neq x_{k l}\right\} \subseteq \mu\left\{(k, l) \in \mathbb{N} \times \mathbb{N}: x_{k l} \notin B_{u}^{p, q}\right\} .
$$

Hence $\mu\left(\left\{(k, l) \in \mathbb{N} \times \mathbb{N}: y_{k l} \neq x_{k l}\right\}\right)=0$. Thus, in the space $\left(X,\|\cdot, \cdot\|_{\infty}\right), y_{k l}=x_{k l}$ for a.a. $(k, l)$. Suppose that $\left\{u_{1}, u_{2}, \ldots, u_{d}\right\}$ is the basis for $(X,\|\cdot, \cdot\|)$. Since $\lim _{m n \rightarrow \infty}\left\|y_{k l}-A\right\|_{\infty}=0$ and $\left\|y_{k l}-A, u_{i}\right\| \leq\left\|y_{k l}-A\right\|_{\infty}$ for all $1 \leq i \leq d, \lim _{m n \rightarrow \infty}\left\|y_{k l}-A, z\right\|_{\infty}=0$ for every $z \in X$. This completes the proof.

In order to prove the equivalence of Definitions 2.5 and 2.6, we shall find it helpful to use Theorems 2.1 and 2.2.

Theorem 2.3 Let $\mu$ be a two-valued measure on $\mathbb{N} \times \mathbb{N}$ and let $\left\{x_{k l}\right\}_{k l \geq 1}$ be a sequence in a 2-normed space $(X,\|\cdot, \cdot\|)$. The sequence $\left\{x_{k l}\right\}$ is $\mu$-statistically convergent if and only if $\left\{x_{k l}\right\}$ is a $\mu$-statistically Cauchy sequence.

\section{New double sequence spaces}

We first state an inequality which will be used throughout this paper: If $\left\{p_{k l}\right\}$ is a bounded double sequence of non-negative real numbers and $\sup _{k, l \in \mathbb{N}} p_{k l}=H$ and $D=\operatorname{Max}\left\{1,2^{H-1}\right\}$, then

$$
\left|a_{k l}+b_{k l}\right|^{p_{k l}} \leq D\left\{\left|a_{k l}\right|^{p_{k l}}+\left|b_{k l}\right|^{p_{k l}}\right\}
$$

for all $k, l$ and $a_{k l}, b_{k l} \in \mathbb{C}$, the set of all complex numbers. Also,

$$
|a|^{p_{k l}} \leq \operatorname{Max}\left\{1,|a|^{H}\right\}
$$

for all $a \in \mathbb{C}$.

By a lacunary sequence $\theta=\left(k_{r}\right) ; r=0,1,2, \ldots$, where $k_{0}=0$, we shall mean an increasing sequence of non-negative integers with $k_{r}-k_{r-1}$ as $r \rightarrow \infty$. The intervals determined by $\theta$ will be denoted by $I_{r}=\left(k_{r-1}, k_{r}\right]$ and $h_{r}=k_{r}-k_{r-1}$. The ratio $\frac{k_{r}}{k_{r-1}}$ will be denoted by $q_{r}$.

Definition 3.1 The double sequence $\theta_{r, s}=\left\{\left(k_{r}, l_{s}\right)\right\}$ is called double lacunary if there exist two increasing sequences of integers such that

$$
k_{0}=0, \quad h_{r}=k_{r}-k_{k-1} \rightarrow \infty \quad \text { as } r \rightarrow \infty
$$

and

$$
l_{0}=0, \quad \bar{h}_{s}=l_{s}-l_{s-1} \rightarrow \infty \quad \text { as } s \rightarrow \infty .
$$

Let us denote $k_{r, s}=k_{r} l_{s}, h_{r, s}=h_{r} \bar{h}_{s}$ and $\theta_{r, s}$ is determined by $I_{r, s}=\left\{(k, l): k_{r-1}<k \leq\right.$ $\left.k_{r} \& l_{s-1}<l \leq l_{s}\right\}, q_{r}=\frac{k_{r}}{k_{r-1}}, \bar{q}_{s}=\frac{l_{s}}{l_{s-1}}$, and $q_{r, s}=q_{r} \bar{q}_{s}$.

Definition 3.2 Suppose that as before $\mu$ is a two-valued measure on $\mathbb{N} \times \mathbb{N}$ and let $M$ be an Orlicz function and $(X,\|\cdot, \cdot\|)$ be a 2 -normed space. Further, let $p=\left\{p_{k l}\right\}$ be a bounded 
sequence of positive real numbers. Now we introduce the following different types of sequence spaces, for all $\epsilon>0$,

$$
\begin{aligned}
W^{\mu} & \left(\theta^{2}, M, \Delta^{m}, p,\|\cdot, \cdot\|\right) \\
= & \left\{x \in S^{\prime \prime}(2-X): \mu\left((k, l) \in \mathbb{N} \times \mathbb{N}: \frac{1}{h_{r s}} \sum_{(k, l) \in I_{r s}}\left[M\left(\left\|\frac{\Delta^{m} x_{k l}-L}{\rho}, z\right\|\right)\right]^{p_{k l}} \geq \epsilon\right)=0\right. \\
& \text { for some } \rho>0 \text { and } L \in X \text { and each } z \in X\}, \\
W_{0}^{\mu}\left(\theta^{2}, M, \Delta^{m}, p,\|\cdot, \cdot\|\right) & \left\{x \in S^{\prime \prime}(2-X): \mu\left((k, l) \in \mathbb{N} \times \mathbb{N}: \frac{1}{h_{r s}} \sum_{(k, l) \in I_{r s}}\left[M\left(\left\|\frac{\Delta^{m} x_{k l}}{\rho}, z\right\|\right)\right]^{p_{k l}} \geq \epsilon\right)=0\right. \\
= & \text { for some } \rho>0 \text { and each } z \in X\}, \\
W_{\infty}^{\mu} & \left(\theta^{2}, M, \Delta^{m}, p,\|\cdot, \cdot\|\right) \\
= & \left\{x \in S^{\prime \prime}(2-X): \exists k>0, \mu(\{(k, l) \in \mathbb{N} \times \mathbb{N}:\right. \\
& \left.\left.\left.\frac{1}{h_{r s}} \sum_{(k, l) \in I_{r s}}\left[M\left(\left\|\frac{\Delta^{m} x_{k l}}{\rho}, z\right\|\right)\right]^{p_{k l}} \geq k\right\}\right)=0 \text { for some } \rho>0 \text { and each } z \in X\right\},
\end{aligned}
$$

where $\Delta^{m} x_{k l}=\Delta^{m-1} x_{k l}-\Delta^{m-1} x_{k+1, l}-\Delta^{m-1} x_{k, l+1}+\Delta^{m-1} x_{k+1, l+1}$.

We now prove the following theorem.

Theorem 3.1 $W^{\mu}\left(\theta, M, \Delta^{m}, p,\|\cdot, \cdot\|\right), W_{0}^{\mu}\left(\theta^{2}, M, \Delta^{m}, p,\|\cdot, \cdot\|\right)$ and $W_{\infty}^{\mu}\left(\theta^{2}, M, \Delta^{m}, p,\|\cdot, \cdot\|\right)$ are linear spaces.

Proof We shall prove the theorem for $W_{0}^{\mu}\left(\theta^{2}, M, \Delta^{m}, p,\|\cdot, \cdot\|\right)$ and others can be proved similarly. Let $\epsilon>0$ be given. Assume that $x, y \in^{2} W_{0}^{\mu}\left(\theta, M, \Delta^{m}, p,\|\cdot, \cdot\|\right)$ and $\alpha, \beta \in \mathbb{R}$, where $x=\left\{x_{k l}\right\}$ and $y=\left\{y_{k l}\right\}$. Further let $z \in X$. Then

$$
\mu\left(\left\{(k, l) \in \mathbb{N} \times \mathbb{N}: \frac{1}{h_{r s}} \sum_{(k, l) \in I_{r s}}\left[M\left(\left\|\frac{\Delta^{m} x_{k l}}{\rho_{1}}, z\right\|\right)\right]^{p_{k l}} \geq \epsilon\right\}\right)=0
$$

for some $\rho_{1}>0$ and

$$
\mu\left(\left\{(k, l) \in \mathbb{N} \times \mathbb{N}: \frac{1}{h_{r s}} \sum_{(k, l) \in I_{r s}}\left[M\left(\left\|\frac{\Delta^{m} y_{k l}}{\rho_{2}}, z\right\|\right)\right]^{p_{k l}} \geq \epsilon\right\}\right)=0
$$

for some $\rho_{2}>0$.

Since $\|\cdot, \cdot\|$ is 2 -normed, $\Delta^{m}$ is linear, therefore the following inequality holds:

$$
\begin{aligned}
& \frac{1}{h_{r s}} \sum_{(k, l, j) \in I_{r s}}\left[M\left(\left\|\frac{\Delta^{m}\left(\alpha x_{k l}+\beta y_{k l}\right)}{|\alpha| \rho_{1}+|\beta| \rho_{2}}, z\right\|\right)\right]^{p_{k l}} \\
& \quad \leq D \frac{1}{h_{r s}} \sum_{(k, l) \in I_{r s}}\left[\frac{|\alpha|}{|\alpha| \rho_{1}+|\beta| \rho_{2}} M\left(\left\|\frac{\Delta^{m} x_{k l}}{\rho_{1}}, z\right\|\right)\right]^{p_{s t}}
\end{aligned}
$$




$$
\begin{aligned}
& +D \frac{1}{h_{r s}} \sum_{(k, l) \in I_{k l}}\left[\frac{|\beta|}{|\alpha| \rho_{1}+|\beta| \rho_{2}} M\left(\left\|\frac{\Delta^{m} y_{k l}}{\rho_{2}}, z\right\|\right)\right]^{p_{k l}} \\
\leq & D F \frac{1}{h_{r s}} \sum_{(k, l) \in I_{r s}}\left[M\left(\left\|\frac{\Delta^{m} x_{k l}}{\rho_{1}}, z\right\|\right)\right]^{p_{k l}}+D F \frac{1}{h_{r s}} \sum_{(k, l) \in I_{r s}}\left[M\left(\left\|\frac{\Delta^{m} y_{k l}}{\rho_{2}}, z\right\|\right)\right]^{p_{k l}},
\end{aligned}
$$

where

$$
F=\operatorname{Max}\left\{1,\left[\frac{|\alpha|}{|\alpha| \rho_{1}+|\beta| \rho_{2}}\right]^{H},\left[\frac{|\beta|}{|\alpha| \rho_{1}+|\beta| \rho_{2}}\right]^{H}\right\} .
$$

From the above inequality we get

$$
\begin{gathered}
\left\{(k, l) \in \mathbb{N} \times \mathbb{N}: \frac{1}{h_{r s}} \sum_{(k, l) \in I_{r s}}\left[M\left(\left\|\frac{\Delta^{m}\left(\alpha x_{k l}+\beta y_{k l}\right)}{|\alpha| \rho_{1}+|\beta| \rho_{2}}, z\right\|\right)\right]^{p_{k l}} \geq \epsilon\right\} \\
\subseteq\left\{(k, l) \in \mathbb{N} \times \mathbb{N}: D F \frac{1}{h_{r s}} \sum_{(k, l) \in I_{r s}}\left[M\left(\left\|\frac{\Delta^{m} x_{k l}}{\rho_{1}}, z\right\|\right)\right]^{p_{k l}} \geq \frac{\epsilon}{2}\right\} \\
\cup\left\{(k, l) \in \mathbb{N} \times \mathbb{N}: D F \frac{1}{h_{r s}} \sum_{(k, l) \in I_{r s}}\left[M\left(\left\|\frac{\Delta^{m} y_{k l}}{\rho_{2}}, z\right\|\right)\right]^{p_{k l}} \geq \frac{\epsilon}{2}\right\} .
\end{gathered}
$$

Hence from (3.1) and (3.2) the required result is proved.

Theorem 3.2 For any fixed $(k, l) \in \mathbb{N} \times \mathbb{N}, W_{\infty}^{\mu}\left(\theta^{2}, M, \Delta^{m}, p,\|\cdot, \cdot\|\right)$ is a paranormed space with respect to the paranorm $g_{k l}: X \rightarrow \mathbb{R}$, defined by

$$
\begin{aligned}
g_{k l}(x)= & \inf _{z \in X} \sum_{(k, l) \in I_{r s}}\left\|x_{k l}, z\right\| \\
& +\inf \left\{\rho^{p_{k l} / H}: \rho>0 \text { s.t. } \sup _{(k, l) \in \mathbb{N} \times \mathbb{N}}\left[M\left(\left\|\frac{\Delta^{m} x_{k l}}{\rho}, z\right\|\right)\right]^{p_{k l}} \leq 1, \forall z \in X\right\} .
\end{aligned}
$$

Proof This can be proved by using the techniques similar to those used in Theorem 4.3 in [18].

Theorem 3.3 Let $M, M_{1}, M_{2}$ be Orlicz functions. Then

(i) $W_{0}^{\mu}\left(\theta^{2}, M_{1}, \Delta^{m}, p,\|\cdot, \cdot\|\right) \subseteq W_{0}^{\mu}\left(\theta^{2}, \operatorname{Mo} M_{1}, \Delta^{m}, p,\|\cdot, \cdot\|\right)$, provided $\left\{p_{k l}\right\}_{k, l \in \mathbb{N} \times \mathbb{N}}$ are such that $H_{0}=\inf p_{k l}>0$;

(ii) $W_{0}^{\mu}\left(\theta^{2}, M_{1}, \Delta^{m}, p,\|\cdot, \cdot\|\right) \cap W_{0}^{\mu}\left(\theta^{2}, M_{2}, \Delta^{m}, p,\|\cdot, \cdot\|\right) \subseteq W_{0}^{\mu}\left(\theta^{2}, M_{1}+M_{2}, \Delta^{m}, p,\|\cdot, \cdot\|\right)$.

Proof (i) Let $\epsilon>0$ be given. Choose $\epsilon_{0}>0$ such that $\max \left\{\epsilon_{0}^{H}, \epsilon_{0}^{H_{0}}\right\}<\epsilon$. Now, using the continuity of $M$, choose $0<\delta<1$ such that $0<t<\delta$ implies that $M(t)<\epsilon_{0}$. Let $\left\{x_{k l}\right\} \in$ $W_{0}^{\mu}\left(\theta^{2}, M_{1}, \triangle^{m}, p,\|\cdot \cdot \cdot\|\right)$. Now, from the definition $\mu(A(\delta))=0$, where

$$
A(\delta)=\left\{(k, l) \in \mathbb{N} \times \mathbb{N}: \frac{1}{h_{r s}} \sum_{(k, l) \in I_{r s}}\left[M_{1}\left(\left\|\frac{\Delta^{m} x_{k l}}{\rho}, z\right\|\right)\right]^{p_{k l}} \geq \delta^{H}\right\} .
$$

Thus if $(k, l) \notin A(\delta)$, then

$$
\frac{1}{h_{r s}} \sum_{(k, l) \in I_{r s}}\left[M_{1}\left(\left\|\frac{\Delta^{m} x_{k l}}{\rho}, z\right\|\right)\right]^{p_{k l}}<\delta^{H},
$$


i.e.,

$$
\sum_{(r, s) \in I_{r s}}\left[M_{1}\left(\left\|\frac{\Delta^{m} x_{k l}}{\rho}, z\right\|\right)\right]^{p_{k l}}<h_{r s} \delta^{H}
$$

i.e.,

$$
\left[M_{1}\left(\left\|\frac{\Delta^{m} x_{k l}}{\rho}, z\right\|\right)\right]^{p_{k l}}<\delta^{H}
$$

for all $(k, l) \in I_{r s}$. Hence

$$
\left[M_{1}\left(\left\|\frac{\Delta^{m} x_{k l}}{\rho}, z\right\|\right)\right]<\delta
$$

for all $(k, l) \in I_{r s}$.

Hence, from the above, using the continuity of $M$, we must have

$$
M\left(\left[M_{1}\left(\left\|\frac{\Delta^{m} x_{k l}}{\rho}, z\right\|\right)\right]\right)<\epsilon_{0}
$$

for all $(k, l) \in I_{r s}$. This implies that

$$
\left[\operatorname{Mo} M_{1}\left(\left\|\frac{\Delta^{m} x_{k l}}{\rho}, z\right\|\right)\right]^{p_{k l}}<\max \left\{\epsilon_{0}^{H}, \epsilon_{0}^{H}\right\}
$$

for all $(k, l) \in I_{r s}$, i.e.,

$$
\sum_{(k, l) \in I_{r s}}\left[\operatorname{Mo} M_{1}\left(\left\|\frac{\Delta^{m} x_{k l}}{\rho}, z\right\|\right)\right]^{p_{k l}}<h_{r s} \max \left\{\epsilon_{0}^{H}, \epsilon_{0}^{H}\right\}<h_{r s} \epsilon,
$$

which again implies that

$$
\frac{1}{h_{r s}} \sum_{(r, s) \in I_{r s}}\left[\operatorname{Mo} M_{1}\left(\left\|\frac{\Delta^{m} x_{k l}}{\rho}, z\right\|\right)\right]^{p_{k l}}<\epsilon
$$

This shows that

$$
\left\{(k, l) \in \mathbb{N} \times \mathbb{N}: \frac{1}{h_{r s}} \sum_{(k, l) \in I_{r s}}\left[\operatorname{Mo} M_{1}\left(\left\|\frac{\Delta^{m} x_{k l}}{\rho}, z\right\|\right)\right]^{p_{k l}} \geq \epsilon\right\} \subseteq A(\delta) .
$$

Therefore

$$
\mu\left(\left\{(k, l) \in \mathbb{N} \times \mathbb{N}: \frac{1}{h_{r s}} \sum_{(k, l) \in I_{r s}}\left[\operatorname{Mo} M_{1}\left(\left\|\frac{\Delta^{m} x_{k l}}{\rho}, z\right\|\right)\right]^{p_{k l}} \geq \epsilon\right\}\right)=0 .
$$

Thus

$$
\left\{x_{k l}\right\} \in W_{0}^{\mu}\left(\theta^{2}, M_{1}, \Delta^{m}, p,\|\cdot, \cdot\|\right) .
$$


Savaş Journal of Inequalities and Applications 2013, 2013:347

Page 9 of 11

http://www.journalofinequalitiesandapplications.com/content/2013/1/347

(ii) Let $\left\{x_{k l}\right\} \in W_{0}^{\mu}\left(\theta^{2}, M_{1}, \Delta^{m}, p,\|\cdot, \cdot\|\right) \cap W_{0}^{\mu}\left(\theta^{2}, M_{2}, \Delta^{m}, p,\|\cdot, \cdot\|\right)$. Then the fact that

$$
\begin{aligned}
\frac{1}{h_{r s}} & {\left[\left(M_{1}+M_{2}\right)\left(\left\|\frac{\Delta^{m} x_{k l}}{\rho}, z\right\|\right)\right]^{p_{k l}} } \\
& \leq \frac{D}{h_{r s}}\left[M_{1}\left(\left\|\frac{\Delta^{m} x_{k l}}{\rho}, z\right\|\right)\right]^{p_{k l}}+\frac{D}{h_{r s}}\left[M_{2}\left(\left\|\frac{\Delta^{m} x_{k l}}{\rho}, z\right\|\right)\right]^{p_{k l}}
\end{aligned}
$$

gives us the result. Hence this completes the proof of the theorem.

Finally we conclude this paper by stating the following theorem.

Theorem 3.4 Let $X\left(\triangle^{m-1}\right), m \geq 1$, stand for $W^{\mu}\left(\theta^{2}, M, \Delta^{m-1}, p,\|\cdot, \cdot\|\right)$ or $W_{0}^{\mu}\left(\theta^{2}, M, \Delta^{m-1}\right.$, $p,\|\cdot, \cdot\|)$ or $W_{\infty}^{\mu}\left(\theta^{2}, M, \Delta^{m-1}, p,\|\cdot, \cdot\|\right)$. Then $X\left(\Delta^{m-1}\right) \varsubsetneqq X\left(\triangle^{m}\right)$. In general, $X\left(\Delta^{i}\right) \varsubsetneqq X\left(\Delta^{m}\right)$ for all $i=1,2,3, \ldots, m-1$.

Proof We shall give the proof for $W_{0}^{\mu}\left(\theta^{2}, M, \Delta^{m-1}, p,\|\cdot, \cdot\|\right)$ only. It can be proved in a simlar way for $W^{\mu}\left(\theta^{2}, M, \Delta^{m-1}, p,\|\cdot, \cdot\|\right)$ and ${ }^{2} W_{\infty}^{\mu}\left(\theta, M, \Delta^{m-1}, p,\|\cdot, \cdot\|\right)$.

Let $x=\left\{x_{k l}\right\}_{k, l \in \mathbb{N}} \in W_{0}^{\mu}\left(\theta^{2}, M, \Delta^{m-1}, p,\|\cdot, \cdot\|\right)$. Let also $\epsilon>0$ be given. Then

$$
\mu\left(\left\{(k, l) \in \mathbb{N} \times \mathbb{N}: \frac{1}{h_{r s}} \sum_{(k, l) \in I_{r s}}\left[M\left(\left\|\frac{\Delta^{m-1} x_{k l}}{\rho}, z\right\|\right)\right]^{p_{k l}} \geq \epsilon\right\}\right)=0
$$

for some $\rho>0$. Since $M$ is non-decreasing and convex, it follows that

$$
\begin{aligned}
\frac{1}{h_{r s}} \sum_{(k, l) \in I_{r s}}\left[M\left(\left\|\frac{\Delta^{m} x_{k l}}{4 \rho}, z\right\|\right)\right]^{p_{k l}} \\
=\frac{1}{h_{r s}} \sum_{(k, l) \in I_{r s}}\left[M\left(\left\|\frac{\Delta^{m-1} x_{k+1, l+1}-\Delta^{m-1} x_{k+1, l}-\Delta^{m-1} x_{k, l+1}+\Delta^{m-1} x_{k l}}{4 \rho}, z\right\|\right)\right]^{p_{k l}} \\
\leq \frac{D^{2}}{h_{r s}} \sum_{(k, l) \in I_{r s}}\left(\left[\frac{1}{4} M\left(\left\|\frac{\Delta^{m-1} x_{k+1, l+1}}{\rho}, z\right\|\right)\right]^{p_{k l}}+\left[\frac{1}{4} M\left(\left\|\frac{\Delta^{m-1} x_{k+1, l}}{\rho}, z\right\|\right)\right]^{p_{k l}}\right. \\
\left.+\left[\frac{1}{4} M\left(\left\|\frac{\Delta^{m-1} x_{k, l+1}}{\rho}, z\right\|\right)\right]^{p_{k l}}+\left[\frac{1}{4} M\left(\left\|\frac{\Delta^{m-1} x_{k, l}}{\rho}, z\right\|\right)\right]^{p_{k l}}\right) \\
\leq \frac{D^{2} G}{h_{r s}} \sum_{(k, l) \in I_{r s}}\left(\left[M\left(\left\|\frac{\Delta^{m-1} x_{k+1, l+1}}{\rho}, z\right\|\right)\right]^{p_{k l}}+\left[M\left(\left\|\frac{\Delta^{m-1} x_{k+1, l}}{\rho}, z\right\|\right)\right]^{p_{k l}}\right. \\
\left.\quad+\left[M\left(\left\|\frac{\Delta^{m-1} x_{k, l+1}}{\rho}, z\right\|\right)\right]^{p_{k l}}+\left[M\left(\left\|\frac{\Delta^{m-1} x_{k, l}}{\rho}, z\right\|\right)\right]^{p_{k l}}\right),
\end{aligned}
$$

where $G=\operatorname{Max}\left\{1,\left(\frac{1}{4}\right)^{H}\right\}$. Hence we have

$$
\begin{aligned}
& \left\{(k, l) \in \mathbb{N} \times \mathbb{N}: \frac{1}{h_{r s}} \sum_{(k, l) \in I_{r s}}\left[M\left(\left\|\frac{\Delta^{m} x_{k l}}{4 \rho}, z\right\|\right)\right]^{p_{k l}} \geq \epsilon\right\} \\
& \subseteq\left\{(k, l) \in \mathbb{N} \times \mathbb{N}: \frac{D^{2} G}{h_{r s}} \sum_{(k, l) \in I_{r s}}\left[M\left(\left\|\frac{\Delta^{m-1} x_{k+1, l+1}}{\rho}, z\right\|\right)\right]^{p_{k l}} \geq \frac{\epsilon}{4}\right\} \\
& \cup\left\{(k, l) \in \mathbb{N} \times \mathbb{N}: \frac{D^{2} G}{h_{r s}} \sum_{(k, l) \in I_{r s}}\left[M\left(\left\|\frac{\Delta^{m-1} x_{k+1, l}}{\rho}, z\right\|\right)\right]^{p_{k l}} \geq \frac{\epsilon}{4}\right\}
\end{aligned}
$$




$$
\begin{aligned}
& \cup\left\{(k, l) \in \mathbb{N} \times \mathbb{N}: \frac{D^{2} G}{h_{r s}} \sum_{(k, l) \in I_{r s}}\left[M\left(\left\|\frac{\Delta^{m-1} x_{k, l+1}}{\rho}, z\right\|\right)\right]^{p_{k l}} \geq \frac{\epsilon}{4}\right\} \\
& \cup\left\{(k, l) \in \mathbb{N} \times \mathbb{N}: \frac{D^{2} G}{h_{r s}} \sum_{(k, l) \in I_{r s}}\left[M\left(\left\|\frac{\Delta^{m-1} x_{k, l}}{\rho}, z\right\|\right)\right]^{p_{k l}} \geq \frac{\epsilon}{4}\right\} .
\end{aligned}
$$

Using (3.3) we get

$$
\mu\left(\left\{(k, l) \in \mathbb{N} \times \mathbb{N}: \frac{1}{h_{r s}} \sum_{(k, l) \in I_{r s}}\left[M\left(\left\|\frac{\Delta^{m} x_{k l}}{4 \rho}, z\right\|\right)\right]^{p_{k l}} \geq \epsilon\right\}\right)=0 .
$$

Therefore $x=\left\{x_{k l}\right\} \in W_{0}^{\mu}\left(\theta^{2}, M, \Delta^{m}, p,\|\cdot, \cdot\|\right)$. This completes the proof.

\section{Competing interests}

The author declares that they have no competing interests.

\section{Received: 16 November 2012 Accepted: 3 July 2013 Published: 26 July 2013}

\section{References}

1. Connor, J: Two valued measure and summability. Analysis 10, 373-385 (1990)

2. Connor, J: R-Type summability methods, Cauchy criterion, P-sets and statistical convergence. Proc. Am. Math. Soc. 115(2), 319-327 (1992)

3. Fast, H: Sur la convergence statistique. Colloq. Math. 2, 241-244 (1951)

4. Fridy, JA: On statistical convergence. Analysis 5, 301-313 (1985)

5. Šalát, T: On statistically convergent sequences of real numbers. Math. Slovaca 30, 139-150 (1980)

6. Móricz, F: Statistical convergence of multiple sequences. Arch. Math. 81, 82-89 (2003)

7. Muresaleen, M, Edely, OHH: Statistical convergence of double sequences. J. Math. Anal. Appl. 288, 223-231 (2003)

8. Savaş, E: On generalized statistical convergence in random 2-normed space. Iran. J. Sci. Technol., Trans. A, Sci. 36(4), 417-423 (2012)

9. Das, P, Malik, P, Savaş, E: On statistical limit point of double sequences. Appl. Math. Comput. 215(3), 499-504 (2009)

10. Patterson, RF, Savaş, E: Lacunary statistical convergence of double sequences. Math. Commun. 10, 55-61 (2000)

11. Savaş, E: On $\bar{\lambda}$-statistically convergent double sequences of fuzzy numbers. J. Inequal. Appl. 2008, Article ID 147827 (2008)

12. Savaș, E, Patterson, RF: $(\lambda, \sigma)$-Double sequence spaces via Orlicz function. J. Comput. Anal. Appl. 10(1), 101-111 (2008)

13. Savaş, E, Patterson, RF: Double sequence spaces defined by Orlicz functions. Iran. J. Sci. Technol., Trans. A, Sci. 31(2), 183-188 (2007)

14. Savaş, E, Mohiuddine, SA: $\bar{\lambda}$-Statistically convergent double sequences in probabilistic normed spaces. Math. Slovaca 62(1), 99-108 (2012)

15. Savaş, E: Some new double sequence spaces defined by Orlicz function in n-normed space. J. Inequal. Appl. 2011, 99 (2011)

16. Savaş, E: On lacunary double statistical convergence in locally solid Riesz spaces. J. Inequal. Appl. 2013, 99 (2013)

17. Das, P, Bhunia, S: Two valued measure and summability of double sequences. Czechoslov. Math. J. 59(134), 1141-1155 (2009)

18. Das, P, Savaş, E, Bhunia, S: Two valued measure and some new double sequence spaces in 2-normed spaces. Czechoslov. Math. J. 61(136), 809-825 (2010)

19. Lindenstrauss, J, Tzafriri, L: On Orlicz sequence spaces. Isr. J. Math. 10, 379-390 (1971)

20. Krasnoselskii, MA, Rutisky, YB: Convex Functions and Orlicz Spaces. Noordhoff, Groningen (1961)

21. Kamthan, PK, Gupta, M: Sequence Spaces and Series. Dekker, New York (1981)

22. Gahler, S: 2-Metrische Räume und ihre topologische Struktur. Math. Nachr. 26, 115-148 (1963)

23. Gahler, S: 2-Normed spaces. Math. Nachr. 28, 1-43 (1964)

24. Gahler, S, Siddiqi, AH, Gupta, SC: Contributions to non-Archimedian functional analysis. Math. Nachr. 69, 162-171 (1975)

25. Gurdal, M, Pehlivan, S: Statistical convergence in 2-normed spaces. Southeast Asian Bull. Math. 33(5), 257-264 (2009)

26. Gurdal, M, Sahiner, A, Acik, I: Approximation theory in 2-Banach spaces. Nonlinear Anal. 71, 1654-1661 (2009)

27. Sahiner, A, Gurdal, M, Saltan, S, Gunawan, H: Ideal convergence in 2-normed spaces. Taiwan. J. Math. 11(5), 1477-1484 (2007)

28. Savaş, E: $\Delta^{m}$-Strongly summable sequences spaces in 2-normed spaces defined by ideal convergence and an Orlicz function. Appl. Math. Comput. 217, 271-276 (2010)

29. Savaş, E: A-Sequence spaces in 2-normed space defined by ideal convergence and an Orlicz function. Abstr. Appl. Anal. 2011, Article ID 741382 (2011)

30. Savaş, E: On some new sequence spaces in 2-normed spaces using ideal convergence and an Orlicz function. J. Inequal. Appl. 2010, Article ID 482392 (2010). doi:10.1155/2010/482392

31. Pringsheim, A: Zur Theorie der zweifach unendlichen Zahlenfolgen. Math. Ann. 53, 289-321 (1900) 
32. Parashar, SD, Choudhary, B: Sequence spaces defined by Orlicz functions. Indian J. Pure Appl. Math. 25(4), 419-428 (1994)

33. Savaş, E: $(A)_{\Delta}(A)$-Double sequence spaces of fuzzy numbers via Orlicz function. Iran. J. Fuzzy Syst. 8(2), 91-103 (2011)

34. Savaș, E, Patterson, RF: Some double lacunary sequence spaces defined by Orlicz functions. Southeast Asian Bull. Math. 35(1), 103-110 (2011)

35. Savaş, E: Some new double sequence spaces defined by Orlicz function in $n$-normed space. J. Inequal. Appl. 2011, Article ID 592840 (2011)

36. Savaş, E: $\bar{\lambda}$-Double sequence spaces of fuzzy real numbers defined by Orlicz function. Math. Commun. 14(2), 287-297 (2009)

37. Savaş, E: On some new double lacunary sequences spaces via Orlicz function. J. Comput. Anal. Appl. 11(3), 423-430 (2009)

38. Savaş, E, Savaş, R: Some $\lambda$-sequence spaces defined by Orlicz functions. Indian J. Pure Appl. Math. 34(12), 1673-1680 (2003)

doi:10.1186/1029-242X-2013-347

Cite this article as: Savaş: On two-valued measure and double statistical convergence in 2-normed spaces. Journal of Inequalities and Applications 2013 2013:347.

Submit your manuscript to a SpringerOpen ${ }^{\circ}$ journal and benefit from:

- Convenient online submission

- Rigorous peer review

- Immediate publication on acceptance

- Open access: articles freely available online

- High visibility within the field

- Retaining the copyright to your article

Submit your next manuscript at $>$ springeropen.com 